\title{
Adverse pregnancy outcomes and later cardiovascular disease
}

Such adverse pregnancy outcomes (APOs) as miscarriage, preterm birth, low-birth weight, gestational diabetes, preeclampsia, and placental abruption have been reported to be associated with an increased risk of later cardiovascular morbidity and mortality. Counseling your patients who have experienced an APO about the protective effect of breastfeeding, eating a heart-healthy diet, exercise, and optimizing weight may help them to live a long and healthy life.

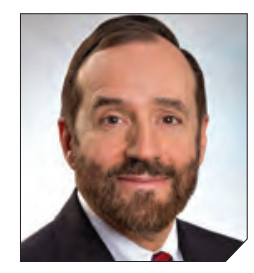

\section{Robert L. Barbieri, MD}

Chair Emeritus, Department of Obstetrics and Gynecology

Interim Chief, Obstetrics

Brigham and Women's Hospital

Kate Macy Ladd Distinguished Professor of Obstetrics,

Gynecology and Reproductive Biology

Harvard Medical School

Boston, Massachusetts

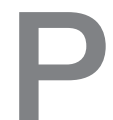

reconception health influences pregnancy outcomes, and in turn, both preconception health and an APO influence adult cardiometabolic health (FIGURE, page 10 ). This editorial is focused on the link between APOs and later cardiometabolic morbidity and mortality, recognizing that preconception health greatly influences the risk of an APO and lifetime cardiometabolic disease.

\section{Adverse pregnancy outcomes}

Major APOs include miscarriage, preterm birth (birth $<37$ weeks' gestation), low birth weight (birthweight $\leq 2,500 \mathrm{~g} ; 5.5 \mathrm{lb}$ ), gestational diabetes (GDM), preeclampsia, and placental abruption. In the United States, among all births, reported rates of the following APOs are: ${ }^{1-3}$

- preterm birth, $10.2 \%$

- low birth weight, $8.3 \%$

doi: 10.12788/obgm.0106
- GDM, $6 \%$

- preeclampsia, $5 \%$

- placental abruption, $1 \%$.

Miscarriage occurs in approximately $10 \%$ to $15 \%$ of pregnancies, influenced by both the age of the woman and the method used to diagnose pregnancy. ${ }^{4}$ Miscarriage, preterm birth, low birth weight, GDM, preeclampsia, and placental abruption have been reported to be associated with an increased risk of later cardiovascular morbidity and mortality.

\section{APOs and cardiovascular disease}

Cardiovascular disease (CVD) affects the majority of people past the age of 60 years and includes 4 major subcategories:

1. coronary heart disease, including myocardial infarction, angina, and heart failure

2. CVD, stroke, and transient ischemic attack
3. peripheral artery disease

4. atherosclerosis of the aorta leading to aortic aneurysm.

Multiple meta-analyses report that APOs are associated with CVD in later life. A comprehensive review reported that the risk of CVD was increased following a pregnancy with one of these APOs: severe preeclampsia (odds ratio [OR], 2.74), GDM (OR, 1.68), preterm birth (OR, 1.93), low birth weight (OR, 1.29), and placental abruption (OR, 1.82). ${ }^{5}$

The link between APOs and CVD may be explained in part by the association of APOs with multiple risk factors for CVD, including chronic hypertension, type 2 diabetes mellitus (T2DM), and dyslipidemia. A meta-analysis of 43 studies reported that, compared with controls, women with a history of preeclampsia have a 3.13 times greater risk of developing chronic hypertension. ${ }^{6}$ Among women with preeclampsia, approximately $20 \%$ 
will develop hypertension within 15 years. ${ }^{7}$ A meta-analysis of 20 studies reported that women with a history of GDM had a 9.51-times greater risk of developing T2DM than women without GDM. ${ }^{8}$ Among women with a history of GDM, over 16 years of follow-up, T2DM was diagnosed in $16.2 \%$, compared with $1.9 \%$ of control women. ${ }^{8}$

\section{CVD prevention - Breastfeeding: An antidote for APOs}

Pregnancy stresses both the cardiovascular and metabolic systems. Breastfeeding is an antidote to the stresses imposed by pregnancy. Breastfeeding women have lower blood glucose $^{9}$ and blood pressure. ${ }^{10}$ Breastfeeding reduces the risk of CVD. In a study of 100,864 parous Australian women, with a mean age of 60 years, ever breastfeeding was associated a lower risk of CVD hospitalization (adjusted hazard ratio [aHR], 0.86; 95\% confidence interval [CI], 0.78-0.96; $P=.005)$ and CVD mortality (aHR, 0.66; 95\% CI, 0.49$0.89 ; P=.006) .{ }^{11}$

\section{CVD prevention-American Heart Association recommendations}

The American Heart Association ${ }^{12}$ recommends lifestyle interventions to reduce the risk of CVD, including:

- Eat a high-quality diet that includes vegetables, fruit, whole grains, beans, legumes, nuts, plant-based protein, lean animal protein, and fish.

- Limit intake of sugary drinks and foods, fatty or processed meats, full-fat dairy products, eggs, highly processed foods, and tropical oils.

- Exercise at least 150 minutes weekly at a moderate activity level,

\section{FIGURE Influencers on adult cardiovascular health}

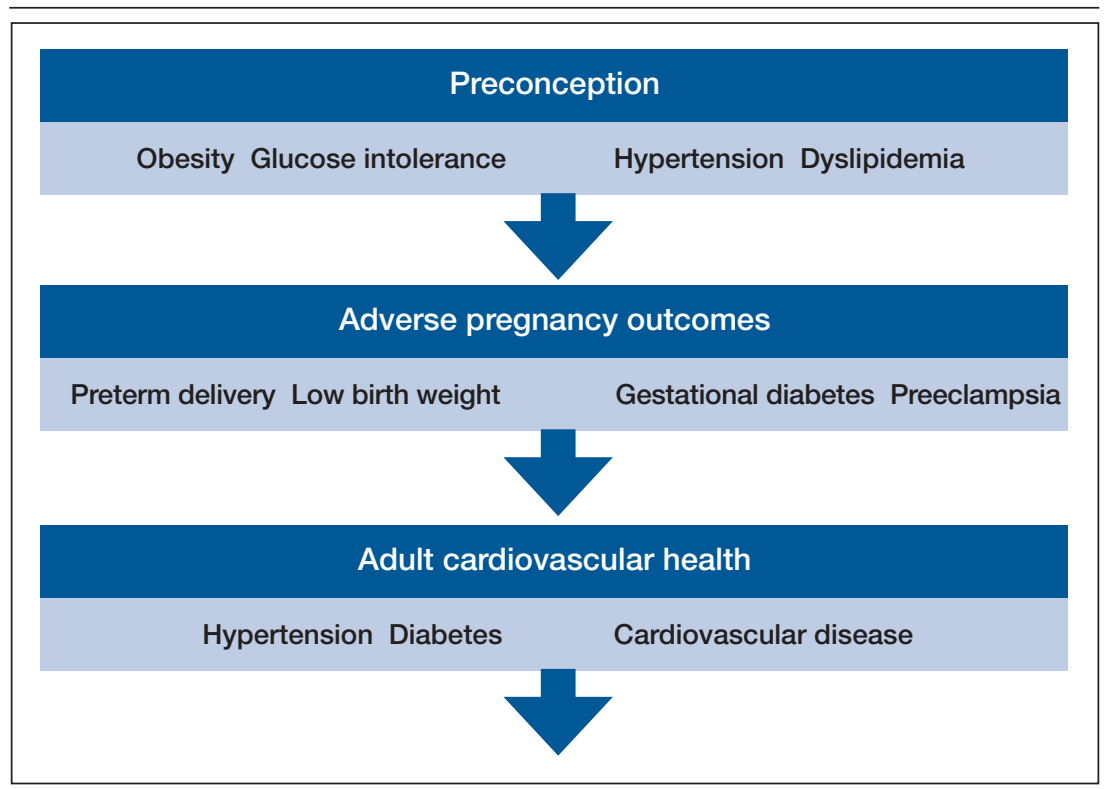

Preconception health influences pregnancy outcomes, and in turn, both preconception health and adverse pregnancy outcomes influence adult cardiometabolic health.

including muscle-strengthening activity.

- Reduce prolonged intervals of sitting.

- Live a tobacco- and nicotine-free life.

- Strive to maintain a normal body mass index.

- Consider using an activity tracker to monitor activity level.

- After 40 years of age calculate CVD risk using a validated calculator such as the American Cardiology Association risk calculator. ${ }^{13}$ This calculator uses age, gender, and lipid and blood pressure measurements to calculate the 10-year risk of atherosclerotic CVD, including coronary death, myocardial infarction, and stroke.

\section{Medications to reduce CVD risk}

Historically, ObGyns have not routinely prescribed medications to treat hypertension, dyslipidemia, or to prevent diabetes. The recent increase in the valuation of return ambulatory visits and a reduction in the valuation assigned to procedural care may provide ObGyn practices the additional resources needed to manage some chronic diseases. Physician assistants and nurse practitioners may help ObGyn practices to manage hypertension, dyslipidemia, and prediabetes.

Prior to initiating a medicine, counseling about healthy living, including smoking cessation, exercise, heart-healthy diet, and achieving an optimal body mass index is warranted.

For treatment of stage II hypertension, defined as blood pressure (BP) measurements with systolic $\mathrm{BP} \geq 140 \mathrm{~mm} \mathrm{Hg}$ and diastolic BP $\geq 90 \mathrm{~mm} \mathrm{Hg}$, therapeutic lifestyle interventions include: optimizing weight, following the DASH diet, restricting dietary sodium, physical activity, and reducing alcohol consumption. Medication treatment 


\section{Miscarriage, placental abruption, and risk of CVD morbidity and mortality}

Miscarriage: To study the association between miscarriage and cardiovascular mortality, investigators with the Nurses' Health Study followed 101,681 women, including 26,102 who reported at least one miscarriage from 1993 to $2017 .{ }^{1}$ During 2,367,839 years of follow-up, before 70 years of age deaths from cancer and cardiovascular disease (CVD) occurred in 2,936 and 269 women, respectively. After controlling for multiple clinical factors, including body mass index, smoking status, physical activity, healthy eating index, gravidity, race, ethnicity, marriage status, history of gestational diabetes mellitus (GDM), hypertensive disorders of pregnancy and family history, there was a significant association between miscarriage and CVD mortality (hazard ratio [HR], 1.48; 95\% confidence interval [Cl], 1.09-1.99). The association was greater for women with 2 (adjusted HR [aHR], 1.82) or 3 (aHR, 1.96) miscarriages than for women with 1 (aHR, 1.40) or no (aHR, 1.00) miscarriages (test for trend, $P=.006$ ). The risk of CVD mortality was greater when the first miscarriage occurred at age $\leq 23$ years $(\mathrm{aHR}, 1.78)$ or 24 to 29 years $(\mathrm{aHR}, 1.59)$ than when the first miscarriage occurred at $\geq 30$ years $(\mathrm{aHR}$ 1.15), referent group of women with no miscarriages (aHR, $1.00)$, test for trend $P=.002$.

There was no significant association between miscarriage and death from cancer ( $\mathrm{HR}, 1.08 ; 95 \% \mathrm{Cl}, 0.94-$ 1.24). There was also no significant association between miscarriage and death from infection, respiratory disease, mental disorders, suicide, accidents, or gastrointestinal disease. There was no association between induced abortion and increased mortality (HR, 1.06; 95\% Cl, 0.70-1.62.) The causal link between miscarriage and CVD mortality is not clear, but a proinflammatory state and endothelial dysfunction may be involved.'

Placental abruption: In a meta-analysis of 7 cohort studies, placental abruption was associated with an increased risk of CVD (HR, 1.82; 95\% Cl, 1.42-2.33). ${ }^{2}$ The reported cohort studies did not control for a history of hypertensive disease of pregnancy, which is a risk factor for both placental abruption and CVD. In a study of 1,555,596 pregnancies in California, 14,881 women $(0.096 \%)$ were identified who received a diagnosis of placental abruption. ${ }^{3}$ Placental abruption was associated with hypertensive disorder of pregnancy, older maternal age, black race, depression, and alcohol and drug misuse. In this study, placental abruption absent a hypertensive disorder of pregnancy was not associated with heart failure $(H R$, 0.90; 95\% Cl, 0.52-1.60). Placental abruption occurring in conjunction with a hypertensive disorder of pregnancy was associated with an increased risk of heart failure $(\mathrm{HR}$, 1.79; 95\% Cl, 1.30-2.48). Absent a hypertensive disorder, placental abruption may not be linked to later CVD. In this study, placental abruption was associated with an increased risk of heart failure after a median follow-up of approximately 5 years (HR, 1.44; 95\% Cl, 1.09-1.90). ${ }^{3}$

\section{References}

1. Wang YX, Minguez-Alarcon L, Gaskins AJ, et al. Association of spontaneous abortion with all cause and cause specific premature mortality: prospective cohort study. BMJ. 2021;372:n530. doi: 10.1136/bmj.n530.

2. Grandi SM, Filion KB, Yoon SA, et al. Circulation. 2019;139:1069-1079. doi:

10.1161/CIRCULATIONAHA.118.036748.

3. DesJardin JY, Healy MJ, Nah G, et al. Placental abruption as a risk factor for heart failure. Am J Cardiol. 2020;131:17-22. doi: 10.1016/j. amjcard.2020.06.034 for essential hypertension is guided by the magnitude of $\mathrm{BP}$ reduction needed to achieve normotension. For women with hypertension needing antihypertensive medication and planning another pregnancy in the near future, labetalol or extendedrelease nifedipine may be first-line medications. For women who have completed their families or who have no immediate plans for pregnancy, an angiotensin-converting enzyme inhibitor, angiotensin receptor blocker, calcium channel blocker, or thiazide diuretic are commonly prescribed. ${ }^{14}$

For the treatment of elevated lowdensity lipoprotein (LDL) cholesterol in women who have not had a cardiovascular event, statin therapy is often warranted when both the LDL cholesterol is $>100 \mathrm{mg} / \mathrm{dL}$ and the woman has a calculated 10-year risk of $>10 \%$ for a cardiovascular event using the American Heart Association or American College of Cardiology calculator. Most women who meet these criteria will be older than age 40 years and many will be under the care of an internal medicine or family medicine specialist, limiting the role of the ObGyn. ${ }^{15-17}$

For prevention of diabetes in women with a history of GDM, both weight loss and metformin (1,750 mg daily) have been shown in clinical trials to reduce the risk of developing T2DM. ${ }^{18}$ Among 350 women with a history of GDM who were followed for 10 years, metformin $850 \mathrm{mg}$ twice daily reduced the risk of developing T2DM by $40 \%$ compared with placebo. ${ }^{19}$ In the same study, lifestyle changes without metformin, including loss of $7 \%$ of body weight plus 150 minutes of exercise weekly was associated with a $35 \%$ reduction in the risk of developing T2DM. ${ }^{19}$ Metformin is one of the least expensive prescription medications and is costeffective for the prevention of T2DM. ${ }^{18}$

Low-dose aspirin treatment for the prevention of CVD in women who have not had a cardiovascular 
event must balance a modest reduction in cardiovascular events with a small increased risk of bleeding events. The US Preventive Services Task Force (USPSTF) recommends low-dose aspirin for a limited group of women, those aged 50 to 59 years of age with a 10-year risk of a cardiovascular event $>10 \%$ who are willing to take aspirin for 10 years. The USPSTF concluded that there is insufficient evidence to recommend low-dose aspirin prevention of CVD in women aged $<50$ years. ${ }^{20}$

\section{Beyond the fourth trimester}

The fourth trimester is the 12-week period following birth. At the comprehensive postpartum visit, the American College of Obstetricians and Gynecologists (ACOG) recommends

\section{References}

1. Martin JA, Hamilton BE, Osterman MJ, et al Births: final data for 2019. Natl Vital Stat Rep. 2021;70:1-51.

2. Deputy NP, Kim SY, Conrey EJ, et al. Prevalence and changes in preexisting diabetes and gestational diabetes among women who had a live birth-United States, 2012-2016. MMWR Morb Mortal Wkly Rep. 2018;67:1201-1207. doi: 10.15585/mmwr.mm6743a2.

3. Fingar KR, Mabry-Hernandez I, Ngo-Metzger Q, et al. Delivery hospitalizations involving preeclampsia and eclampsia, 2005-2014. Statistical brief \#222. In: Healthcare Cost and Utilization Project (HCUP) Statistical Briefs [Internet]. Agency for Healthcare Research and Quality: Rockville, MD; April 2017.

4. Magnus MC, Wilcox AJ, Morken NH, et al. Role of maternal age and pregnancy history in risk of miscarriage: prospective register-based study. $B M J$. 2019;364:869.

5. Parikh NI, Gonzalez JM, Anderson CAM, et al. Adverse pregnancy outcomes and cardiovascular disease risk: unique opportunities for cardiovascular disease prevention in women. Circulation. 2021;143:e902-e916. doi: 10.1161 /CIR.0000000000000961.

6. Brown MC, Best KE, Pearce MS, et al. Cardiovascular disease risk in women with pre-eclampsia: systematic review and meta-analysis. Eur J Epidemiol. 2013;28:1-19. doi: 10.1007/s10654-0139762-6.

7. Groenfol TK, Zoet GA, Franx A, et al; on behalf of the PREVENT Group. Trajectory of cardiovascular risk factors after hypertensive disorders of pregnancy. Hypertension. 2019;73:171-178. doi: that women with APOs be counseled about their increased lifetime risk of maternal cardiometabolic disease. ${ }^{21}$ In addition, ACOG recommends that at this visit the clinician who will assume primary responsibility for the woman's ongoing medical care in her primary medical home be clarified. One option is to ensure a high-quality hand-off to an internal medicine or family medicine clinician. Another option is for a clinician in the ObGyn's office practice, including a physician assistant, nurse practitioner, or office-based ObGyn, to assume some role in the primary care of the woman.

\section{An APO is not only a pregnancy problem}

An APO reverberates across a woman's
10.1161/HYPERTENSIONAHA.118.11726.

8. Vounzoulaki E, Khunti K, Abner SC, et al. Progression to type 2 diabetes in women with a known history of gestational diabetes: systematic review and meta-analysis. BMJ. 2020;369:ml361. doi: 10.1136/bmj.m1361.

9. Tarrant M, Chooniedass R, Fan HSL, et al. Breastfeeding and postpartum glucose regulation among women with prior gestational diabetes: a systematic review. J Hum Lact. 2020;36:723-738. doi: 10.1177/0890334420950259.

10. Park S, Choi NK. Breastfeeding and maternal hypertension. Am J Hypertens. 2018;31:615-621. doi: 10.1093/ajh/hpx219.

11. Nguyen B, Gale J, Nassar N, et al. Breastfeeding and cardiovascular disease hospitalization and mortality in parous women: evidence from a large Australian cohort study. J Am Heart Assoc. 2019;8:e011056. doi: 10.1161/JAHA.118.011056.

12. Eight things you can do to prevent heart disease and stroke. American Heart Association website. https://www.heart.org/en/healthy-living /healthy-lifestyle/prevent-heart-disease-andstroke. Last Reviewed March 14, 2019. Accessed May 19, 2021

13. ASCVD risk estimator plus. American College of Cardiology website. https://tools.acc.org /ascvd-risk-estimator-plus/\#!/calculate /estimate/. Accessed May 19, 2021.

14. Ferdinand KC, Nasser SA. Management of essential hypertension. Cardiol Clin. 2017;35:231-246. doi: 10.1016/j.ccl.2016.12.005.

15. Packard CJ. LDL cholesterol: how low to go? Trends Cardiovasc Med. 2018;28:348-354. doi: 10.1016/j.tcm.2017.12.011. lifetime, increasing the risk of CVD and diabetes. In the United States the mean age at first birth is 27 years. ${ }^{1}$ The mean life expectancy of US women is 81 years. ${ }^{22}$ Following a birth complicated by an APO there are 5 decades of opportunity to improve health through lifestyle changes and medication treatment of obesity, hypertension, dyslipidemia, and hyperglycemia, thereby reducing the risk of CVD.

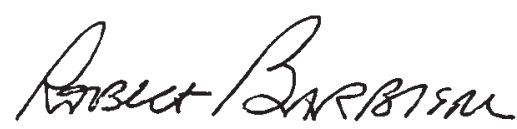

RBARBIERI@MDEDGE.COM

Dr. Barbieri reports no financial relationships relevant to this article.

16. Simons L. An updated review of lipid-modifying therapy. Med J Aust. 2019;211:87-92. doi: 10.5694 /mja2.50142.

17. Chou R, Dana T, Blazina I, et al. Statins for the prevention of cardiovascular disease in adults: evidence report and systematic review for the US Preventive Services Task Force. JAMA. 2016;316:2008. doi: 10.1001/jama.2015.15629.

18. Moin T, Schmittdiel JA, Flory JH, et al. Review of metformin use for type 2 diabetes mellitus prevention. Am J Prev Med. 2018;55:565-574. doi: 10.1016/j.amepre.2018.04.038.

19. Aorda VR, Christophi CA, Edelstein SL, et al, for the Diabetes Prevention Program Research Group. The effect of lifestyle intervention and metformin on preventing or delaying diabetes among women with and without gestational diabetes: the Diabetes Prevention Program outcomes study 10-year follow-up. J Clin Endocrinol Metab. 2015;100:16461653. doi: 10.1210/jc.2014-3761.

20. Bibbins-Domingo K, U.S. Preventive Services Task Force. Aspirin use of the primary prevention of cardiovascular disease and colorectal cancer: U.S. Preventive Services Task Force Recommendation Statement. Ann Int Med. 2016; 164: 836-845. doi: 10.7326/M16-0577.

21. American College of Obstetricians and Gynecologists. ACOG Committee Opinion No. 736: optimizing postpartum care. Obstet Gynecol. 2018;131:e140-e150. doi: 10.1097 /AOG.0000000000002633.

22. National Center for Health Statistics. Health, United States, 2017: Table 015. Hyattsville, MD; 2021. https://www.cdc.gov/nchs/data /hus/2017/015.pdf. Accessed May 18, 2021. 